\title{
A Turbo SDMA Receiver for Strongly Nonlinearly Distorted MC-CDMA Signals
}

\author{
Paulo Silva \\ ISR-IST/EST, Univ. of Algarve \\ Portugal \\ Email:psilva@ualg.pt
}

\author{
Rui Dinis \\ ISR-IST, Tech. Univ. of Lisbon \\ Portugal \\ Email: rdinis@ist.utl.pt
}

\begin{abstract}
In this paper we consider the use of SDMA (Space Division Multiple Access) techniques for the uplink of MCCDMA systems (MultiCarrier - Coded Division Multiple Access) where the transmitted signals face strong nonlinear distortion effects. We define an iterative receiver that jointly performs a turbo-MUD (MultiUser Detection) as well as estimation and cancellation of the nonlinear distortion effects. ${ }^{1}$
\end{abstract}

\section{INTRODUCTION}

SDMA (Space Division Multiple Access) techniques employ multiple antennas to increase substantially the system capacity [1]. In this paper we consider the use of SDMA techniques for the uplink of MC-CDMA systems (MultiCarrier - Coded Division Multiple Access). As with other multicarrier schemes, MC-CDMA signals have strong envelope fluctuations and high PMEPR values (Peak-to-Mean Envelope Power Ratio), which lead to amplification difficulties. For this reason, it is desirable to reduce the envelope fluctuations of the transmitted signals. This is particularly important for the uplink transmission, since an efficient, low-cost power amplification is desirable at the MT (Mobile Terminal). Several techniques have been recommended for reducing the envelope fluctuations of multicarrier signals (see [6] and references within). A promising approach is to employ clipping techniques, combined with a frequency-domain filtering so as to reduce the envelope fluctuations of the transmitted signals while maintaining the spectral occupation of conventional schemes [6]. However, the nonlinear distortion effects can be severe when a low-PMEPR transmission is intended [6], [7].

As with other CDMA schemes, since the transmission over time-dispersive channels destroys the orthogonality between users, an FDE (Frequency-Domain Equalizer) is required before the despreading operation [2]. To avoid significant noise enhancement for channels with deep in-band notches, the FDE is usually optimized under an MMSE criterion (Minimum Mean-Squared Error) [2]. However, an FDE/MMSE does not perform an ideal channel inversion and we are not able to fully orthogonalize the different spreading codes of an MC-CDMA signal. This means that we can have severe interference levels, especially for fully loaded systems and/or when different powers are assigned to different spreading codes. To improve

\footnotetext{
${ }^{1}$ This work was partially supported by the FCT project POSI/CPS/46701/2002 - MC-CDMA, the FCT/POCI 2010 research grant SFRH / BD / 24520 / 2005 and the European IST project C-MOBILE.
}

the performance several turbo-MUD receivers were proposed for conventional CDMA systems [3], [4], as well as MCCDMA [5]. A promissing tecvhnique for MC-CDMA with nonlinear transmitters was proposded in [8], where nonlinear distortion effects are iteratively estimated and compensated. However, for low SNR (Signal-to-Noise Ratio) the error decisions might lead to error propagation effects, since errors in the estimation of nonlinear distortion effects, precluding an efficient cancellation. This is particularly serious for high system load and/or when no space diversity is used [8]. This is especially important when the spreading factor is small and/or if we decrease the clipping level, to reduce further the PMEPR of the transmitted signals.

To reduce error propagation effects in the typical region of operation we use channel decoder outputs in the feedback loop, in a turbo-like fashion (a similar approach was proposed for OFDM schemes [9]). We define an iterative receiver that jointly performs a turbo-MUD as well as estimation and cancellation of nonlinear distortion effects, taking in account the distortion's frequency distribution that is inherent to the transmitted signals.

\section{SYSTEM CHARACTERIZATION}

We consider the uplink transmission of MC-CDMA signals employing frequency-domain spreading. We have an SDMA architecture, corresponding to a MIMO (MultipleInput, Multiple-Output) system with $P$ users (MTs), transmitting independent data blocks, and $L$ receive antennas at the BS (Base Station). It is assumed that each MT has a single transmit antenna. The frequency-domain block to be transmitted by the $p$ th $\mathrm{MT}$ is $\left\{S_{k, p} ; k=0,1, \ldots, N-1\right\}$, where $N=K M$, with $K$ denoting the spreading factor and $M$ the number of data symbols for that MT. The frequencydomain symbols are given by $S_{k, p}=\xi_{p} C_{k, p} A_{k \operatorname{Mod} M, p}$, ( $x$ Mod $y$ is the reminder of the division of $x$ by $y$ ) where $\xi_{p}$ is an appropriate weighting coefficient that accounts for the propagation losses, $\left\{A_{k, p} ; k=0,1, \ldots, M-1\right\}$ is the block of data symbols associated to the $p$ th MT and $\left\{C_{k, p} ; k=0,1, \ldots, N-1\right\}$ is the corresponding spreading sequence $^{2}$ (a pseudo-random spreading is assumed, with $C_{k, p}$

\footnotetext{
${ }^{2}$ This corresponds to uniformly spread the chips associated to a given symbol within the transmission band, i.e., to employ a rectangular interleaver with dimensions $K \times M$.
} 
belonging to a QPSK constellation; without loss of generality, it is assumed that $\left|C_{k, p}\right|=1$ ). The transmitter structure is based on the nonlinear signal processing schemes proposed in [6] for reducing the PMEPR of OFDM signals. It is shown in [7] that the frequency-domain block to be transmitted by the $p$ th MT $\left\{S_{k, p}^{T x}=S_{k, p}^{C} G_{k} ; k=0,1, \ldots, N^{\prime}-1\right\}$ can be decomposed into useful and nonlinear self-interference components: $S_{k, p}^{T x}=\alpha_{p} S_{k, p} G_{k}+D_{k, p} G_{k}$, where $G_{k}, k=$ $0,1, \ldots, N^{\prime}-1$, are the frequency-domain filtering coefficients, in order to reduce the out-of-band radiation levels inherent to the nonlinear operation, and $\alpha_{p}$ defined in [6], [7]. Throughout this paper we assume that $G_{k}=1$ for the $N$ in-band subcarriers and 0 for the $N^{\prime}-N$ out-of-band subcarriers. In this case $S_{k, p}^{T x}=\alpha_{p} S_{k, p}+D_{k, p}$, for the $N$ in-band subcarriers and 0 otherwise. It can be shown that $D_{k, p}$ is approximately Gaussian-distributed, with zero mean; moreover, $E\left[D_{k, p} D_{k^{\prime}, p}^{*}\right]$ can be computed analytically, as described in [6], [7].

\section{RECEIVER STRUCTURE}

\section{A. Linear Transmitters}

As usual, it is assumed that the length of the CP (Cyclic Prefix) is higher than the length of the overall channel impulse response. The received time-domain block associated to the $l$ th diversity branch, after discarding the samples associated to the $\mathrm{CP}$, is $\left\{y_{n}^{(l)} ; n=0,1, \ldots, N-1\right\}$. The corresponding frequency-domain block (i.e., the length- $N$ DFT (Discrete Fourier Transform) of the block $\left\{y_{n}^{(l)} ; n=0,1, \ldots, N-1\right\}$ ) is $\left\{Y_{k}^{(l)} ; k=0,1, \ldots, N-1\right\}$.

Let us consider first a linear transmitter. In this case, the frequency-domain block transmitted by the $p$ th $\mathrm{MT}$ is $\left\{S_{k, p}^{T x}=\right.$ $\left.S_{k, p} ; k=0,1, \ldots, N^{\prime}-1\right\}$ and

$$
\begin{aligned}
Y_{k}^{(l)} & =\sum_{p=1}^{P} S_{k, p} H_{k, p}^{C h(l)}+N_{k}^{(l)}= \\
& =\sum_{p=1}^{P} A_{k \operatorname{Mod} M, p} C_{k, p} \xi_{p} H_{k, p}^{C h(l)}+N_{k}^{(l)}= \\
& =\sum_{p=1}^{P} A_{k \operatorname{Mod} M, p} H_{k, p}^{(l)}+N_{k}^{(l)}
\end{aligned}
$$

with $H_{k, p}^{C h(l)}$ denoting the channel frequency response between the $p$ th MT and the $l$ th diversity branch, at the $k$ th subcarrier, $N_{k}^{(l)}$ the corresponding channel noise and $H_{k, p}^{(l)}=$ $\xi_{p} H_{k, p}^{C h(l)} C_{k, p}$. To detect the $k$ th symbol of the $p$ th MT we will use the set of subcarriers $\Psi_{k}=\{k, k+M, \ldots, k+(K-1) M\}$.

By defining $\mathbf{Y}(k)=\left[\mathbf{Y}^{(1)}(k) \cdots \mathbf{Y}^{(L)}(k)\right]^{T}$, with $\mathbf{Y}^{(l)}(k)$ denoting the line vector with the received samples associated to the set of frequencies $\Psi_{k}$, for the $l$ th antenna, and $\mathbf{A}(k)=$ $\left[A_{k \operatorname{Mod} M, 1} \ldots A_{k \operatorname{Mod} M, P}\right]^{T}$, we have

$$
\mathbf{Y}(k)=\mathbf{H}^{T}(k) \mathbf{A}(k)+\mathbf{N}(k)
$$

$\left((\cdot)^{T}\right.$ denote the transpose matrix), where $\mathbf{N}(k)=$ $\left[\mathbf{N}^{(1)}(k) \cdots \mathbf{N}^{(L)}(k)\right]^{T}$, with $\mathbf{N}^{(l)}(k)$ denoting the line vector with the noise samples associated to the set of frequencies $\Psi_{k}$, for the lth antenna. In (2), $\mathbf{H}(k)$ is the overall channel matrix associated to $\mathbf{A}(k)$, i.e., $\mathbf{H}(k)=\left[\mathbf{H}^{(1)}(k) \cdots \mathbf{H}^{(L)}(k)\right]$, with $\mathbf{H}^{(l)}(k)$ denoting a $(P \times K)$ matrix, with lines associated to the different MTs and columns associated to the set of frequencies $\Psi_{k}$, for the $l$ th antenna.

This receiver can be regarded as an iterative multiuser detector with PIC (Parallel Interference Cancelation). The receiver can be described as follows. For a given iteration, the detection of $\mathbf{A}(k)$ employs $L$ feedforward filters (one for each receive antennas) and $P$ feedback loops. The feedforward filters are designed to minimize the MAI (Multiple Access Interference) that cannot be cancelled by the feedback loops. For the first iteration we do not have any information about the MT's symbols and the receiver reduces to a linear multiuser receiver.

For each iteration, the samples associated to $\mathbf{A}(k), \tilde{\mathbf{A}}(k)$ are given by

$$
\tilde{\mathbf{A}}(k)=\mathbf{F}^{T}(k) \mathbf{Y}(k)-\mathbf{B}^{T}(k) \overline{\mathbf{A}}(k)
$$

where $\tilde{\mathbf{A}}(k)$ is defined as $\mathbf{A}(k), \mathbf{F}(k)=\left[\mathbf{F}^{(1)}(k) \cdots \mathbf{F}^{(L)}(k)\right]^{T}$ is the matrix of the feedforward filters' coefficients, with $\mathbf{F}^{(l)}(k)$ denoting a $(P \times K)$ matrix, with lines associated to the different MTs and columns associated to the set of frequencies $\Psi_{k}$, for the $l$ th antenna, and $\mathbf{B}(k)$ is a $P \times P$ matrix with the feedback filters' coefficients. $\overline{\mathbf{A}}(k)$, also defined as $\mathbf{A}(k)$, is the "soft decision" of $\mathbf{A}(k)$ from the SISO (Soft-In, Soft-Out) channel decoder, from the previous iteration. The SISO block, that can be implemented as defined in [10], provides the LLRs (LogLikelihood Ratios) of both the "information bits" and the "coded bits". The input of the SISO block are LLRs of the "coded bits" at the multiuser detector.

In [8] it is shown that, assuming that the transmitted symbols are selected from a QPSK constellation under a Gray mapping rule (the generalization to other cases is straightforward), $\bar{A}_{k, p}$ is given by

$$
\bar{A}_{k, p}=\tanh \left(\frac{L_{k, p}^{I}}{2}\right)+j \tanh \left(\frac{L_{k, p}^{Q}}{2}\right)
$$

where $L_{k, p}^{I}=\frac{2}{\sigma_{p}^{2}} \tilde{A}_{k, p}^{I}$ and $L_{k, p}^{Q}=\frac{2}{\sigma_{p}^{2}} \tilde{A}_{k, p}^{Q}$, are the LLRs of the "in-phase bit" and the "quadrature bit", associated to $A_{k, p}^{I}=\operatorname{Re}\left\{A_{k, p}\right\}$ and $A_{k, p}^{Q}=\operatorname{Im}\left\{A_{k, p}\right\}$, respectively, with

$$
\sigma_{p}^{2}=\frac{1}{2} E\left[\left|A_{k, p}-\tilde{A}_{k, p}\right|^{2}\right] \approx \frac{1}{2 M} \sum_{k=0}^{M-1} E\left[\left|\hat{A}_{k, p}-\tilde{A}_{k, p}\right|^{2}\right]
$$

and $\hat{A}_{k, p}$ denoting the "hard decisions" associated to $\tilde{A}_{k, p}$.

The hard decisions $\hat{A}_{k, p}^{I}= \pm 1$ and $\hat{A}_{k, p}^{Q}= \pm 1$ are defined according to the signs of $L_{k, p}^{I}$ and $L_{k, p}^{Q}$, respectively; $\rho_{k, p}^{I}=\tanh \left(\frac{\left|L_{k, p}^{I}\right|}{2}\right)$ and $\rho_{k, p}^{Q}=\tanh \left(\frac{\left|L_{k, p}^{Q}\right|}{2}\right)$ can be regarded as the reliabilities associated to the "in-phase" and "quadrature" bits of the $k$ th symbol of the $p$ th MT. For the first iteration, $\rho_{k, p}^{I}=\rho_{k, p}^{Q}=0$ and $\bar{A}_{k, p}=0$. We can also 
define the blockwise reliability

$$
\rho_{p}=\frac{1}{M} \sum_{k=0}^{M-1} \frac{E\left[A_{k, p}^{*} \hat{A}_{k, p}\right]}{E\left[\left|A_{k, p}\right|^{2}\right]}=\frac{1}{2 M} \sum_{k=0}^{M-1}\left(\rho_{k, p}^{I}+\rho_{k, p}^{Q}\right) .
$$

It can be shown that the optimum feedforward coefficients in the MMSE sense can be written as

$$
\mathbf{F}(k)=\mathbf{F}^{I}(k) \boldsymbol{\Gamma}^{-1}
$$

with $\boldsymbol{\Gamma}=\operatorname{diag}\left(\gamma_{1}, \ldots, \gamma_{P}\right)$, where $\gamma_{p}=$ $\sum_{k^{\prime} \in \Psi_{k}} \sum_{l=1}^{L} F_{k^{\prime}, p}^{(l)} H_{k^{\prime}, p}^{(l)}$ and

$$
\mathbf{F}^{I}(k)=\left[\mathbf{H}^{H}(k)\left(\mathbf{I}_{P}-\mathbf{P}^{2}\right) \mathbf{H}(k)+\beta \mathbf{I}_{K L}\right]^{-1} \mathbf{H}^{H}(k),
$$

$\left((\cdot)^{H}\right.$ denotes the Hermitian matrix and $\mathbf{I}_{X}$ being the $X$ by- $X$ identity matrix $)$, with $\mathbf{P}=\operatorname{diag}\left(\rho_{1}, \ldots, \rho_{P}\right)$ and $\beta=$ $E\left[\left|N_{k}^{(l)}\right|^{2}\right] / E\left[\left|A_{k, p}\right|^{2}\right]$.

The optimum feedback coefficients are given by

$$
\mathbf{B}(k)=\mathbf{H}(k) \mathbf{F}(k)-\mathbf{I}_{P} .
$$

If we do not have data estimates for the different MTs, $\rho_{p}=0(p=1,2, \ldots, P)$, and the feedback coefficients are zero. Therefore, (3) reduces to

$$
\tilde{\mathbf{A}}(k)=\mathbf{F}^{T}(k) \mathbf{Y}(k),
$$

which corresponds to the linear receiver.

It can be shown that the optimum feedforward coefficients can be written in the form

$$
\mathbf{F}(k)=\mathbf{H}^{H}(k) \mathbf{V}(k),
$$

apart a normalization factor as in (7), with $\mathbf{V}(k)$ given by

$$
\mathbf{V}(k)=\left[\left(\mathbf{I}_{P}-\mathbf{P}^{2}\right) \mathbf{H}(k) \mathbf{H}^{H}(k)+\beta \mathbf{I}_{P}\right]^{-1} .
$$

The computation of the feedforward coefficients from (11) is simpler than the direct computation, from (8), especially when $P<K$ and/or $L>1$.

\section{B. Nonlinear Transmitters}

It was shown in [9] that we can improve significantly the performance of OFDM schemes submitted to nonlinear devices by employing a receiver with iterative cancelation of nonlinear distortion effects. This concept can be extended to MC-CDMA, leading to the receiver structure of fig. 1 . The basic idea behind this receiver is to use an estimate of the nonlinear self-distortion $\left\{\bar{D}_{k, p} ; k=0,1, \ldots, N-1\right\}$ provided by the preceding iteration to remove the nonlinear distortion effects in the received samples. Therefore, the received frequency-domain block associated to the $l$ th diversity antenna, $\left\{Y_{k}^{(l)} ; k=0,1, \ldots, N-1\right\}$, is replaced by the corrected block $Y_{k}^{C o r r}(l)=Y_{k}^{(l)}-\sum_{p=1}^{P} H_{k, p}^{C h(l)} D_{k, p}, k=0,1, \ldots, N-1$. The remaining of the receiver is similar, with the optimum feedforward coefficients given by (7), but with

$$
\begin{aligned}
\mathbf{F}^{I}(k) & =\left[\mathbf{H}^{H}(k)\left(\mathbf{I}_{P}-\mathbf{P}^{2}\right) \mathbf{H}(k)+\beta \mathbf{I}_{K L}+\right. \\
+ & \left.\frac{1}{2 \sigma_{A}^{2}} \mathbf{H}^{C h^{H}}(k) \mathbf{R}_{D}(k) \mathbf{H}^{C h}(k)\right]^{-1} \mathbf{H}^{H}(k),
\end{aligned}
$$

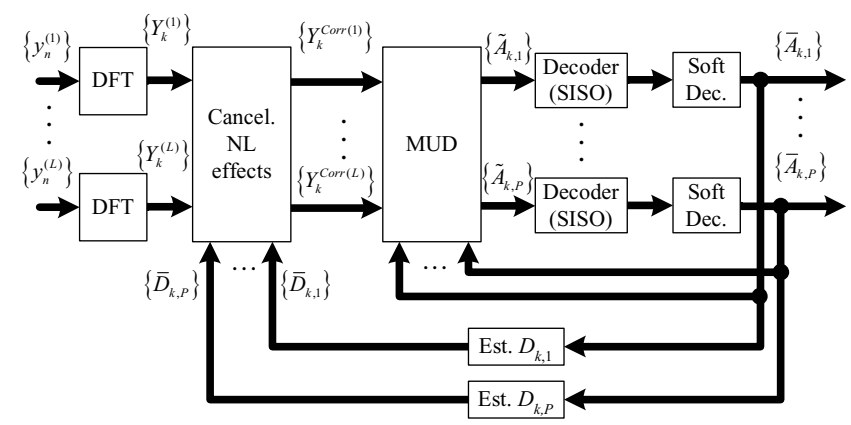

Fig. 1. Iterative receiver with cancelation of nonlinear distortion effects.

where $\mathbf{H}^{C h}(k)$ denote the channel frequency response matrix, defined as $\mathbf{H}(k), \beta=\frac{E\left[\left|N_{k}^{(l)}\right|^{2}\right]}{E\left[\left|\alpha_{p} A_{k, p}\right|^{2}\right]+E\left[\left|D_{k, p}\right|^{2}\right]}$ and $\mathbf{R}_{D}(k)$ is a diagonal matrix with elements $E\left[\left|D_{k^{\prime}, p}-\bar{D}_{k^{\prime}, p}\right|^{2}\right] \approx(1-$ $\left.\rho_{p}^{2}\right) E\left[\left|D_{k, p}\right|^{2}\right], k^{\prime}=k, k+M, \ldots$.

Again, it can be shown that the optimum feedforward coefficients can be written as (11) but, in this case, with

$\mathbf{V}(k)=\left[\left(\mathbf{I}_{P}-\mathbf{P}^{2}\right) \mathbf{H}(k) \mathbf{H}^{H}(k)+\eta \mathbf{H}^{C h}(k) \mathbf{H}^{C h^{H}}(k)+\beta \mathbf{I}_{P}\right]^{-1}$.

For a given iteration, $\left\{\bar{D}_{k, p} ; k=0,1, \ldots, N-1\right\}$ can be estimated from $\left\{\bar{A}_{k, p} ; k=0,1, \ldots, M-1\right\}$ as follows: $\left\{\bar{A}_{k, p} ; k=0,1, \ldots, M-1\right\}$ is re-spread to generate the "average block to be transmitted" $\left\{\bar{S}_{k, p} ; k=0,1, \ldots, N-1\right\}$; $\left\{\bar{S}_{k, p} ; k=0,1, \ldots, N-1\right\}$ is submitted to a replica of the nonlinear signal processing scheme employed in the $p$ th transmitter so as to form the "average transmitted block" $\left\{\bar{S}_{k, p}^{T x} ; k=0,1, \ldots, N-1\right\} ; \bar{D}_{k, p}$ is given by $\bar{D}_{k, p}=$ $\bar{S}_{k, p}^{T x}-\alpha_{p} \bar{S}_{k, p}$ (naturally, for the first iteration, $\bar{D}_{k, p}=0$ ).

\section{Performance Results}

In this section we present a set of performance results concerning the iterative receiver structures proposed in this paper for the uplink of MC-CDMA systems with frequencydomain spreading. The spreading factor is $K=4$ and we have $M=64$ data symbols for each user, corresponding to blocks with length $N=K M=256$, plus an appropriate CP. QPSK constellations, with Gray mapping, are employed. To reduce the envelope fluctuations of the transmitted signals (and the PMEPR) while maintaining the spectral occupation of conventional MC-CDMA schemes, each MT employs the clipping techniques combined with a frequency-domain filtering proposed in [6] (the power amplifiers are assumed to be linear for the (reduced) dynamic range of the envelope fluctuations of the transmitted signals). The receiver (i.e., the BS) knows the characteristics of the PMEPR-reducing signal processing technique employed by each MT.

We consider the power delay profile type $\mathrm{C}$ for the HIPERLAN/2 (HIgh PERformance Local Area Network) [11], with uncorrelated Rayleigh fading for the different MTs and for the different paths. The duration of the useful part of the block is $4 \mu \mathrm{s}$ and the $\mathrm{CP}$ has duration $1.25 \mu \mathrm{s}$. We consider coded BER performances under perfect synchronization 
and channel estimation conditions ${ }^{3}$. We consider the wellknown rate-1/2, 64-state convolutional code with generators $1+D^{2}+D^{3}+D^{5}+D^{6}$ and $1+D+D^{2}+D^{3}+D^{6}$. A random interblock interleaving of the coded bits is assumed before the mapping procedure. The SISO decoder is implemented using the Max-Log-MAP approach. We have $\xi_{p}=1$ for all MTs, i.e., we have a perfect power control. At the BS we have $L=2$ uncorrelated receive antennas, for diversity purposes.

Let us first consider that we have $P=4 \mathrm{MTs}$ and a normalized clipping level, identical to all MTs, of $s_{M} / \sigma=1$. This allows PMEPR values between $1.0 \mathrm{~dB}$ and $4.3 \mathrm{~dB}$, depending on the out-of-band radiation levels (if we want to maintain the spectral occupation of conventional MC-CDMA signals the PMEPR is 4.3dB) [6] (for conventional MC-CDMA signals with a large number of subcarriers the PMEPR is about $8.4 \mathrm{~dB}$ ). Fig. 2 shows the corresponding coded BER performances for each iteration (averaged over all MTs), as well as the performance for a linear transmitter. Clearly, the performance of the linear receiver (first iteration) is very poor, with high irreducible error floors due to the nonlinear distortion effects. This is especially serious for high clipping levels and/or when no space diversity is used $(L=1)$. As we increase the number of iterations improve significantly the performances, that can be close to the ones obtained with linear transmitters.

Let us assume now that we have $P=K L=8 \mathrm{MTs}$, corresponding to a fully loaded scenario and the very low clipping level $s_{M} / \sigma=0.5$ (allowing PMEPR values between $0.5 \mathrm{~dB}$ and $4.1 \mathrm{~dB}$, depending on the out-of-band radiation levels). Fig. 3 shows the corresponding coded BER performances as well as the performance for $s_{M} / \sigma=1$ and for a linear transmitter. Once again, the iterative receiver allows significant performance improvements, even for high system load and/or small clipping levels.

\section{CONCLUSIONS}

In this paper we considered the use of SDMA techniques for the uplink transmission of MC-CDMA signals employing strongly nonlinear transmitters. We proposed an iterative receiver structure that combine turbo-MUD and estimation and cancellation of the nonlinear distortion effects that are inherent to the transmitted signals.

Our performance results showed that the proposed receiver structure allows good performances, close to the linear receiver ones, even for high system load and/or when a low-PMEPR is intended for each MT.

\section{REFERENCES}

[1] R. Gitlin, J. Salz and J. Winters, "The Impact of Antenna Diversity on the Capacity of Wireless Communication Systems", IEEE Trans. on Comm., Vol. 42, No. 2/3/4, 1740-1751, Feb./Mar./Apr. 1994.

[2] H. Sari, "Orthogonal Multicarrier CDMA and its Detection on Frequency-Selective Channels", European Trans. on Telecomm., Vol. 13, No. 5, pp. 439-445, Sep.-Oct. 2002.

${ }^{3}$ It should be noted that perfect time synchronization between the blocks associated to different MTs is not required since some time mismatches can be absorbed by the $\mathrm{CP}$.

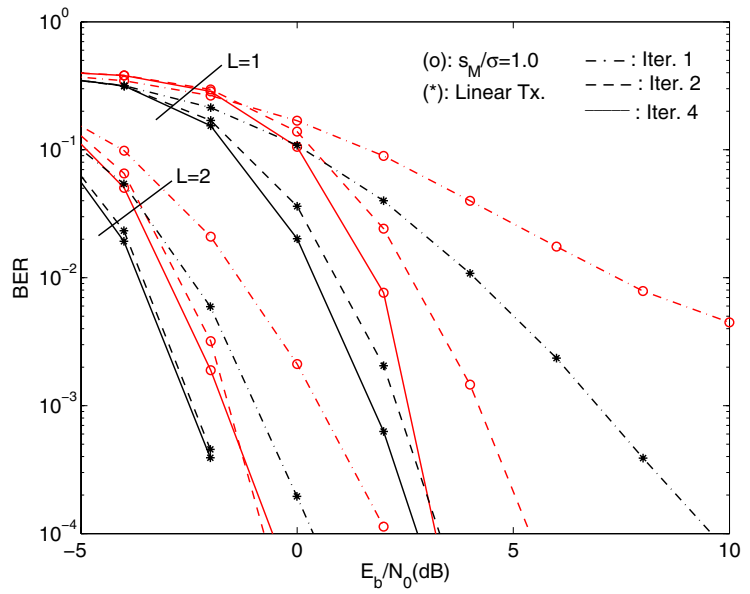

Fig. 2. Average coded BER performance for iterations 1, 2 and 4 (better performances as we increase the number of iterations), for $P=4$ and $L=1$ and 2 , when linear and nonlinear transmitters are considered.

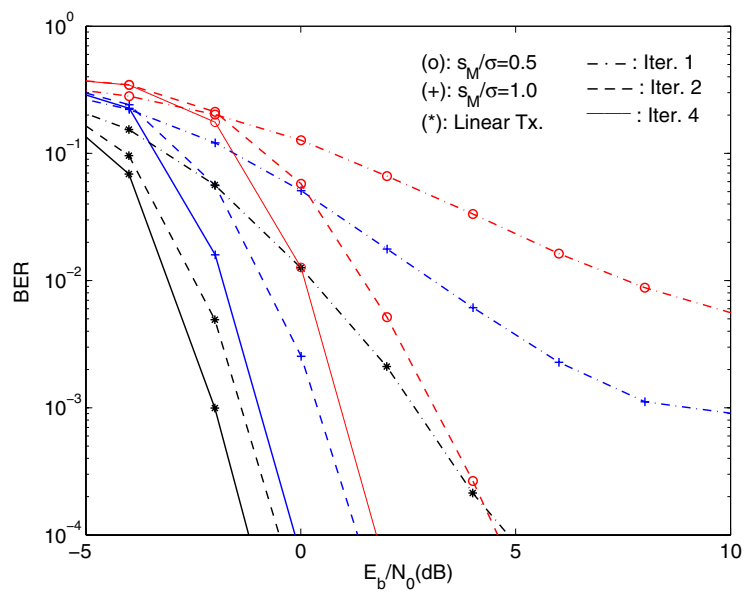

Fig. 3. Average coded BER performance for iterations 1, 2 and 4, for $P=8$ and $L=2$, when linear and nonlinear transmitters are considered.

[3] J. Shen and A. G. Burr, "Iterative Multi-User-Antenna Detector for MIMO CDMA Emploiyng Space-Time Turbo Codes", IEEE GLOBECOM'02, Taipei, Nov. 2002.

[4] X. Wang and H. Poor, "Iterative (Turbo) Soft Interference Cancellation and Decoding for Coded CDMA", IEEE Trans. on Comm., Vol. 47, No. 7, pp. 1046-1061, Jul. 1999.

[5] S. Kaiser and J. Hagenauer, "Multi-Carrier CDMA with Iterative Decoding and Soft-Interference Cancellation", IEEE GLOBECOM'97, Phoenix, Nov. 1997.

[6] R. Dinis and A. Gusmão, "A Class of Nonlinear Signal Processing Schemes for Bandwidth-Efficient OFDM Transmission with Low Envelope Fluctuation", IEEE Trans. on Comm., Vol. 52, No. 11, pp. 2009-2018, Nov. 2004.

[7] R. Dinis and P. Silva, "Analytical Evaluation of Nonlinear Effects in MC-CDMA Signals", IEEE Trans. on Wireless Comm., Vol. 5, No. 8, Aug. 2006.

[8] P. Silva and R. Dinis, "Joint Multiuser Detection and Cancelation of Nonlinear Distortion Effects for the Uplink of MC-CDMA Systems", IEEE PIMRC'06, Helsinki, Sep. 2006.

[9] A. Gusmão, R. Dinis and P. Torres, "Low-PMEPR OFDM Transmission with an Iterative Receiver Technique for Cancellation of Nonlinear Distortion", IEEE VTC05(Fall), Sep. 2005.

[10] B.Vucetic and J.Yuan, Turbo Codes: Principles and Applications, Kluwer Academic Publ., 2002.

[11] ETSI, "Channel models for HIPERLAN/2 in Different Indoor Scenarios”, ETSI EP BRAN 3ERIO85B, pp. 1-8, March 1998. 